\title{
Well-posed results for nonlocal fractional parabolic equation involving Caputo-Fabrizio operator
}

\author{
Tran Thanh Phong, Le Dinh Long* \\ Division of Applied Mathematic, Thu Dau Mot University, Binh Duong province, Viet Nam.
}

\begin{abstract}
In this paper, we study the parabolic problem associated with non-local conditions, with the Caputo-Fabrizio derivative. Equations on the sphere have many important applications in physics, phenomena, and oceanography. The main motivation for us to study non-local boundary value problems comes from two main reasons: the first reason is that current major interest in several application areas. The second reason is to study approximation for the terminal value problem. With some given data, we prove that the problem has only the solution for two cases. In case $\epsilon=0$, we prove the problem has a local solution. In case $\epsilon>0$, then the problem has a global solution. The main tools and techniques in our demonstration are of using Banach's fixed point theorem in conjunction with some Fourier series analysis involved some evaluation of spherical harmonic function. Several upper and lower upper limit techniques for the Mittag-Lefler functions are also applied.
\end{abstract}

Keywords: Nonlocal parabolic equation, Banach fixed point theory, sphere, regularity.

2020 MSC: 35R11, 35B65, 26A33.

(C)2022 All rights reserved.

\section{Introduction}

The partial differential equation on the sphere attracts many mathematicians interested in research. We can present some typical results such as $[8,25-28,33,35]$. These types of equations have applications in geophysics and oceanography. Recently, many authors have been involved in the development of fractional analysis. Its applications often appear in areas such as capacitor theory, engineering, electrode-electrolyte interface models feedback amplifers, medical, [2, 5-7, 24, 34], Jafari and his group $[15,19,30,32]$, Inc and his group [3, 23, 36], and Hammouch and her group [17, 20, 21]. Due to the application needs of different models, many hierarchical derivative operators arise. Some related problems fraction operators have proposed the theory of integral and derivative of non-fractions back to Letnikov, Leibniz, Riemann, Liouville, and Caputo. As we observe, there are a number of these operators that possess power multiplication and thus cause limitations in modeling physical problems. To mitigate and overcome these shortcomings, an alternative fractionation operator multiplied by exponential decay was introduced by Caputo and Fabrizio [9]. The most prominent among the fractional derivatives is the Caputo derivative. This is the derivative that contains the singularity kernels. In [9], the authors invented

\footnotetext{
*Corresponding author

Email addresses: phongtt.khtn@tdmu.edu.vn (Tran Thanh Phong), ledinhlong@tdmu.edu.vn (Le Dinh Long)
}

doi: $10.22436 /$ jmcs.026.04.04

Received: 2021-09-18 Revised: 2021-10-19 Accepted: 2021-11-05 
the Caputo-Fabrizio fractional derivative with the purpose of avoiding singular kernels. In recent times, several mathematicians have been interested in the development and simulation of the Caputo-Fabrizio Derivative, which is an operator that has been widely applied in many fields, such as biology, physics, control systems, materials science, and dynamics, [4], besides, its applications are also shown in some of the following documents $[1,11-14,16,18,22,31]$.

In this paper, we consider parabolic equation with Caputo-Fabrizio derivative on the unit sphere $S^{n} \subset R^{n+1}$ as follows

$$
\begin{cases}{ }_{C F} D_{t}^{\gamma} u-\Delta^{*} u=F(u(x, t)), & (x, t) \text { in } S^{n} \times(0, T), \\ u(x, 0)+\beta u(x, T)=f(x), & x \text { in } S^{n},\end{cases}
$$

where ${ }_{C F} \mathrm{D}_{t}^{\gamma}$ is Caputo-Fabrizio operator for fractional derivatives which is defined as (see [29])

$$
{ }_{C F} D_{t}^{\gamma} v(t)=\frac{H(\alpha)}{1-\alpha} \int_{0}^{t} \mathcal{D}_{\gamma}(t-v) \frac{\partial v(v)}{\partial v} d v, \text { for } t \geqslant 0
$$

where we denote by the kernel $\mathcal{D}_{\alpha}(z)=\exp \left(-\frac{\alpha}{1-\alpha} z\right)$ and $H(\alpha)$ satisfies $H(0)=H(1)=1$, (see, e.g., $[9,10])$. The main contributions of the article are clearly shown as follows.

- The first result of the paper is related to the existence of local solutions of problem (1.1) when $\epsilon>0$. Additionally, we also provide proof of the existence of global solution to problem (1.1) when $\epsilon=0$.

- The second result is the convergence of the solution of problem (1.1) when $\epsilon>0$ to the solution of the problem when $\epsilon=0$.

This article is organized as follows. Section 2 provides some preliminary on spherical harmonics and the definitions of the mild solution. In Section 2, we present our main results including two main theorems. Finally, the proof of some theorems is completed in Section 4.

\section{Preliminaries}

Let's briefly introduce some background knowledge about spherical harmonics. Spherical harmonics are polynomials which satisfy $\Delta_{\chi} Y(x)=0$ (where $\Delta_{x}$ is the Laplacian operator in $R^{n+1}$ ) and are restricted to the surface of the Euclidean sphere $S^{n}$. The eigenvalues for $-\Delta$ are

$$
\lambda_{\ell}=\ell(\ell+n-1), \quad \ell=0,1,2, \ldots
$$

and the respective eigenfunctions are the spherical harmonics $Y_{\ell}(x)$ of order $\ell$, i.e.,

$$
\Delta Y_{\ell}(x)=-\lambda_{\ell} Y_{\ell}(x)
$$

The space of all spherical harmonics of degree $\ell$ on $S^{n}$, denoted by $V_{\ell}$, has an orthonormal basis

$$
\left\{Y_{\ell k}(x): k=1,2,3, \ldots, N(n, \ell)\right\},
$$

where

$$
N(n, 0)=1, \quad N(n, \ell)=\frac{(2 \ell+n-1) \Gamma(\ell+n-1)}{\Gamma(l+1) \Gamma(n)}, \ell \geqslant 1 .
$$

For any $f \in L^{2}\left(S^{n}\right)$, we can express it in terms of spherical harmonics as follows

$$
f=\sum_{\ell=0}^{\infty} \sum_{k=1}^{N(n, \ell)} \widehat{f}_{\ell k} Y_{\ell k}, \widehat{f}_{\ell k}=\int_{S^{n}} f \bar{Y}_{\ell k} d S
$$


where $d S$ is the surface measure of the unit sphere. The Sobolev space $\mathrm{H}^{\sigma}\left(\mathrm{S}^{n}\right)$ with real parameter $\sigma$ consists of all distributions $f$ such that

$$
\|f\|_{H^{\sigma}\left(S^{n}\right)}^{2}=\sum_{\ell=0}^{\infty} \sum_{k=1}^{N(n, \ell)}\left(1+\lambda_{\ell}\right)^{\sigma}\left|\widehat{f}_{\ell k}\right|^{2}<\infty
$$

Obviously, the norm stems from an inner product

$$
<\mathrm{f}, \mathrm{g}>_{\mathrm{H}^{\sigma}}=\sum_{\ell=0}^{\infty} \sum_{k=1}^{\mathrm{N}(\mathrm{n}, \ell)}\left(1+\lambda_{\ell}\right)^{\sigma} \widehat{\mathrm{f}}_{\ell k} \widehat{\mathrm{g}}_{\ell k}
$$

\section{Well-posed results for nonlocal value problem}

In this section, we focus on the nonlocal value problem

$$
\begin{cases}{ }_{C} D_{t}^{\gamma} u-\Delta^{*} u=F(u(x, t)), & (x, t) \text { in } S^{2} \times(0, T), \\ u(x, 0)+\epsilon u(x, T)=f(x), & x \text { in } S^{2},\end{cases}
$$

In order to study further about the solution of the problem (3.1) with $\epsilon>0$, we learn about the problem (3.1) with $\epsilon=0$. If $\epsilon=0$, then we give the following definition.

Definition 3.1. The function $u$ is called a mild solution of problem (1.1) if it satisfies that the following nonlinear equation

$$
u(t)=\mathbf{B}_{\gamma}(t) u_{0}+\int_{0}^{t} \mathbf{B}_{\gamma}(t-r) F(u(r)) d r
$$

where $\mathbf{B}_{\gamma}(\mathbf{t})$ is defined by

$$
\mathbf{B}_{\gamma}(\mathrm{t}) v=\sum_{\ell=0}^{\infty} \sum_{k=1}^{N(n, \ell)} \frac{1}{1+(1-\gamma) \lambda_{\ell}} \exp \left(\frac{-\gamma \lambda_{\ell}}{1+(1-\gamma) \lambda_{\ell}} t\right) \widehat{v}_{\ell k} Y_{\ell k}, \quad v \in \mathrm{L}^{2}\left(\mathrm{~S}^{\mathrm{n}}\right) .
$$

First, we need to prove the result later, and use it to support our future proof. For any $v \in \mathrm{H}^{\sigma-2}\left(\mathrm{~S}^{n}\right)$, we obtain that the following estimate

$$
\left\|\mathbf{B}_{\gamma}(\mathrm{t}) v\right\|_{H^{\sigma}\left(S^{n}\right)} \leqslant \frac{1}{1-\gamma}\|v\|_{H^{\sigma-2}\left(S^{n}\right)} .
$$

Indeed, since the fact that $v \in \mathrm{H}^{\sigma}\left(\mathrm{S}^{n}\right)$, using Parseval's equality, one has

$$
\left\|\mathbf{B}_{\gamma}(\mathrm{t}) v\right\|_{H^{\sigma}\left(S^{n}\right)}^{2}=\sum_{\ell=0}^{\infty} \sum_{k=1}^{N(n, \ell)}\left(1+\lambda_{\ell}\right)^{\sigma}\left(\frac{1}{1+(1-\gamma) \lambda_{\ell}}\right)^{2} \exp \left(\frac{-2 \gamma \lambda_{\ell}}{1+(1-\gamma) \lambda_{\ell}} t\right)\left|\hat{v}_{\ell k}\right|^{2} .
$$

Due to the fact that

$$
\frac{1}{1+(1-\gamma) \lambda_{\ell}} \leqslant \frac{1}{1-\gamma}\left(1+\lambda_{\ell}\right)^{-1}
$$

we know that

$$
\left\|\mathbf{B}_{\gamma}(\mathrm{t}) v\right\|_{H^{\sigma}\left(S^{n}\right)}^{2} \leqslant\left(\frac{1}{1-\gamma}\right)^{2} \sum_{\ell=0}^{\infty} \sum_{k=1}^{N(n, \ell)}\left(1+\lambda_{\ell}\right)^{\sigma-2}\left|\hat{v}_{\ell k}\right|^{2} .
$$

Therefore, we can find that the following bound

$$
\left\|\mathbf{B}_{\gamma}(\mathrm{t}) v\right\|_{H^{\sigma}\left(S^{n}\right)} \leqslant \frac{1}{1-\gamma}\|v\|_{H^{\sigma-2}\left(S^{n}\right)} .
$$


Theorem 3.2. Let us assume that $\mathrm{F}(0)=0$ and

$$
\left\|\mathrm{F}\left(v_{1}\right)-\mathrm{F}\left(v_{2}\right)\right\|_{\mathrm{H}^{\mathrm{p}}\left(\mathrm{S}^{\mathfrak{n}}\right)} \leqslant \mathrm{K}_{\mathrm{f}}\left\|v_{1}-v_{2}\right\|_{\mathrm{H}^{\mathrm{m}}\left(\mathrm{S}^{\mathfrak{n}}\right)^{\prime}} 0 \leqslant \mathrm{~m} \leqslant \mathrm{p}+2 .
$$

a) Then with $T$ small enough, the problem (3.1) has a unique solution in $\mathrm{u}^{\epsilon} \in \mathrm{L}^{\infty}\left(0, \mathrm{~T} ; \mathrm{H}^{\mathrm{m}}\left(\mathrm{S}^{\mathrm{n}}\right)\right)$.

b) Let $\epsilon=0$. Then for any $\mathrm{T}>0$, the problem (3.1) has a unique solution in $\mathrm{u} \in \mathrm{L}^{\infty}\left(0, \mathrm{~T} ; \mathrm{H}^{\mathrm{m}}\left(\mathrm{S}^{\mathrm{n}}\right)\right)$.

Proof. Let us divide the proof into two part.

Part $a$. Let us assume that problem (3.1) has a unique solution $\mathfrak{u}^{\epsilon}$, then we have

$$
\begin{aligned}
u^{\epsilon}(x, t)= & \sum_{\ell=0}^{\infty} \sum_{k=1}^{N(n, \ell)} \frac{1}{1+(1-\gamma) \lambda_{\ell}} \exp \left(\frac{-\gamma \lambda_{\ell}}{1+(1-\gamma) \lambda_{\ell}} t\right) \widehat{\varphi}_{\ell k} Y_{\ell k}(x) \\
& +\int_{0}^{t}\left[\sum_{\ell=0}^{\infty} \sum_{k=1}^{N(n, \ell)} \frac{1}{1+(1-\gamma) \lambda_{\ell}} \exp \left(\frac{-\gamma \lambda_{\ell}}{1+(1-\gamma) \lambda_{\ell}}(t-r)\right) \widehat{F}_{\ell k}\left(u^{\epsilon}\right)(r) Y_{\ell k}(x)\right] d r,
\end{aligned}
$$

where $u(x, 0)=\varphi(x)$. By letting $t=T$, we find that

$$
\begin{aligned}
\widehat{u}_{\ell k}^{\epsilon}(T)= & \frac{1}{1+(1-\gamma) \lambda_{\ell}} \exp \left(\frac{-\gamma \lambda_{\ell}}{1+(1-\gamma) \lambda_{\ell}} \mathrm{T}\right) \widehat{\varphi}_{\ell k} \\
& +\int_{0}^{T} \frac{1}{1+(1-\gamma) \lambda_{\ell}} \exp \left(\frac{-\gamma \lambda_{\ell}}{1+(1-\gamma) \lambda_{\ell}}(T-r)\right) \widehat{F}_{\ell k}\left(u^{\epsilon}\right)(r) d r .
\end{aligned}
$$

From the condition $u(x, 0)+\epsilon u(x, T)=f(x)$, we obtain that

$$
\epsilon \widehat{u}_{\ell k}(T)+\widehat{\varphi}_{\ell k}=\widehat{f}_{\ell k} \text {. }
$$

This implies that

$$
\widehat{\varphi}_{\ell k}=\frac{\widehat{f}_{\ell k}-\epsilon \int_{0}^{T} \frac{1}{1+(1-\gamma) \lambda_{\ell}} \exp \left(\frac{-\gamma \lambda_{\ell}}{1+(1-\gamma) \lambda_{\ell}}(T-r)\right) \widehat{F}_{\ell k}\left(u^{\epsilon}\right)(r) d r}{1+\frac{\epsilon}{1+(1-\gamma) \lambda_{\ell}} \exp \left(\frac{-\gamma \lambda_{\ell}}{1+(1-\gamma) \lambda_{\ell}} T\right)} .
$$

Combining (3.5) and (3.6), we arrive at

$$
\begin{aligned}
u^{\epsilon}(x, t)= & \sum_{\ell=0}^{\infty} \sum_{k=1}^{N(n, \ell)} \frac{1}{1+(1-\gamma) \lambda_{\ell}} \frac{\exp \left(\frac{-\gamma \lambda_{\ell}}{1+(1-\gamma) \lambda_{\ell}} \mathrm{t}\right)}{1+\frac{\epsilon}{1+(1-\gamma) \lambda_{\ell}} \exp \left(\frac{-\gamma \lambda_{\ell}}{1+(1-\gamma) \lambda_{\ell}} \mathrm{T}\right)} \widehat{\mathrm{f}}_{\ell k} \mathrm{Y}_{\ell k}(\mathrm{x}) \\
& -\epsilon \sum_{\ell=0}^{\infty} \sum_{k=1}^{\mathrm{N}(\mathrm{n}, \ell)} \frac{1}{1+(1-\gamma) \lambda_{\ell}} \frac{\exp \left(\frac{-\gamma \lambda_{\ell}}{1+(1-\gamma) \lambda_{\ell}} \mathrm{t}\right)}{1+\frac{\epsilon}{1+(1-\gamma) \lambda_{\ell}} \exp \left(\frac{-\gamma \lambda_{\ell}}{1+(1-\gamma) \lambda_{\ell}} \mathrm{T}\right)} \\
& \times\left[\int_{0}^{T} \frac{1}{1+(1-\gamma) \lambda_{\ell}} \exp \left(\frac{-\gamma \lambda_{\ell}}{1+(1-\alpha) \lambda_{\ell}}(\mathrm{T}-\mathrm{r})\right) \widehat{\mathrm{F}}_{\ell k}\left(\mathrm{u}^{\epsilon}\right)(\mathrm{r}) \mathrm{dr}\right] \mathrm{Y}_{\ell k}(\mathrm{x}) \\
& +\int_{0}^{\mathrm{t}}\left[\sum_{l=0}^{\infty} \sum_{\mathrm{k}=1}^{\mathrm{N}(\mathrm{n}, \ell)} \frac{1}{1+(1-\gamma) \lambda_{\ell}} \exp \left(\frac{-\gamma \lambda_{\ell}}{1+(1-\gamma) \lambda_{\ell}}(\mathrm{t}-\mathrm{r})\right) \widehat{\mathrm{F}}_{\ell k}\left(\mathrm{u}^{\epsilon}\right)(\mathrm{r}) \mathrm{Y}_{\ell k}(\mathrm{x})\right] \mathrm{dr} .
\end{aligned}
$$

Set the following operator

$$
\mathbf{D}_{\gamma}^{\epsilon}(\mathrm{T}) v=\sum_{\ell=0}^{\infty} \sum_{k=1}^{\mathrm{N}(\mathrm{n}, \ell)} \frac{1}{1+\frac{\epsilon}{1+(1-\gamma) \lambda_{\ell}} \exp \left(\frac{-\gamma \lambda_{\ell}}{1+(1-\gamma) \lambda_{\ell}} \mathrm{T}\right)} \widehat{v}_{\ell k} \mathrm{Y}_{\ell k}(\mathrm{x})
$$


If $v \in \mathrm{H}^{\sigma}\left(\mathrm{S}^{\mathrm{n}}\right)$, then we have

$$
\left\|\mathbf{D}_{\gamma}^{\epsilon}(\mathrm{T}) \nu\right\|_{\mathrm{H}^{\sigma}\left(\mathrm{S}^{\mathrm{n}}\right)}^{2}=\sum_{\ell=0}^{\infty} \sum_{k=1}^{\mathrm{N}(\mathrm{n}, \ell)}\left(1+\lambda_{\ell}\right)^{\sigma}\left(\frac{1}{1+\frac{\epsilon}{1+(1-\gamma) \lambda_{\ell}} \exp \left(\frac{-\gamma \lambda_{\ell}}{1+(1-\gamma) \lambda_{\ell}} \mathrm{T}\right)}\right)^{2}\left|\widehat{v}_{\ell k}\right|^{2} .
$$

Noting that

$$
\begin{aligned}
\left(\frac{1}{1+\frac{\epsilon}{1+(1-\gamma) \lambda_{\ell}} \exp \left(\frac{-\gamma \lambda_{\ell}}{1+(1-\gamma) \lambda_{\ell}} T\right)}\right)^{2} & \leqslant\left(\frac{1+(1-\gamma) \lambda_{\ell}}{\epsilon}\right)^{2} \exp \left(\frac{2 \gamma \lambda_{\ell} T}{1+(1-\gamma) \lambda_{\ell}}\right) \\
& \leqslant \frac{1}{\epsilon^{2}}\left(1+\lambda_{\ell}\right)^{2} \exp \left(\frac{2 \gamma \top}{1-\gamma}\right)\left|\widehat{v}_{\ell k}\right|^{2} .
\end{aligned}
$$

The connection between (3.9) and (3.10) tells us that

$$
\left\|\mathbf{D}_{\gamma}^{\epsilon}(\mathrm{T}) \mathcal{v}\right\|_{H^{\sigma}\left(\mathrm{S}^{n}\right)}^{2} \leqslant \frac{1}{\epsilon^{2}} \exp \left(\frac{2 \gamma \mathrm{T}}{1-\gamma}\right) \sum_{\ell=0}^{\infty} \sum_{k=1}^{N(n, \ell)}\left(1+\lambda_{\ell}\right)^{\sigma+2}\left|\widehat{v}_{\ell k}\right|^{2},
$$

which gives the following bound

$$
\left\|\mathbf{D}_{\gamma}^{\epsilon}(\mathrm{T}) v\right\|_{\mathrm{H}^{\sigma}\left(S^{\mathfrak{n}}\right)} \leqslant \frac{1}{\epsilon} \exp \left(\frac{\gamma \mathrm{T}}{1-\gamma}\right)\|v\|_{H^{\sigma+2}\left(S^{n}\right)} .
$$

From the combinations of (3.2) and (3.8), we can rewrite the solution of (3.7) as the following operator

$$
u^{\epsilon}(x, t)=\mathbf{B}_{\gamma}(t) D_{\gamma}^{\epsilon}(T) f-\epsilon \mathbf{B}_{\gamma}(t) D_{\gamma}^{\epsilon}(T) \int_{0}^{T} \mathbf{B}_{\gamma}(T-r) F\left(u^{\epsilon}(r)\right) d r+\int_{0}^{t} B_{\gamma}(t-r) F\left(u^{\epsilon}(r)\right) d r
$$

Set the following funtion

$$
J(v)(t)=\mathbf{B}_{\gamma}(t) \mathbf{D}_{\gamma}^{\epsilon}(T) f-\epsilon \mathbf{B}_{\gamma}(t) \mathbf{D}_{\gamma}^{\epsilon}(T) \int_{0}^{T} \mathbf{B}_{\gamma}(T-r) F(v(r)) d r+\int_{0}^{t} \mathbf{B}_{\gamma}(t-r) F(v(r)) d r .
$$

If $f \in H^{m}\left(S^{n}\right)$, then using (3.3), we get the following bound

$$
\left\|\mathbf{B}_{\gamma}(\mathrm{t}) \mathbf{D}_{\gamma}^{\epsilon}(\mathrm{T}) \mathrm{f}\right\|_{\mathrm{H}^{\mathrm{m}}\left(\mathrm{S}^{\mathfrak{n}}\right)} \leqslant \frac{1}{1-\gamma}\left\|\mathbf{D}_{\gamma}^{\epsilon}(\mathrm{T}) \mathrm{f}\right\|_{\mathrm{H}^{\mathrm{m}-2}\left(\mathrm{~S}^{\mathfrak{n}}\right)} \leqslant \frac{1}{1-\gamma} \frac{1}{\epsilon} \exp \left(\frac{\gamma \mathrm{T}}{1-\gamma}\right)\|\mathbf{f}\|_{\mathrm{H}^{\mathrm{m}}\left(\mathrm{S}^{\mathfrak{n}}\right)} .
$$

This implies that $\mathrm{J}(v)$ belongs to the space $\mathrm{L}^{\infty}\left(0, \mathrm{~T} ; \mathrm{H}^{\mathrm{m}}\left(\mathrm{S}^{\mathrm{n}}\right)\right)$ if $v=0$. Taking $v_{1}, v_{2} \in \mathrm{L}^{\infty}\left(0, \mathrm{~T} ; \mathrm{H}^{\mathrm{m}}\left(\mathrm{S}^{\mathrm{n}}\right)\right)$, then we get

$$
\begin{aligned}
J\left(v_{1}\right)(t)-J\left(v_{2}\right)(t)= & -\epsilon \mathbf{B}_{\gamma}(t) \mathbf{D}_{\gamma}^{\epsilon}(\mathrm{T}) \int_{0}^{\mathrm{T}} \mathbf{B}_{\gamma}(\mathrm{T}-\mathrm{r})\left(\mathrm{F}\left(v_{1}(\mathrm{r})\right)-\mathrm{F}\left(v_{2}(\mathrm{r})\right)\right) \mathrm{d} r \\
& +\int_{0}^{\mathrm{t}} \mathbf{B}_{\gamma}(\mathrm{t}-\mathrm{r})\left(\mathrm{F}\left(v_{1}(\mathrm{r})\right)-\mathrm{F}\left(v_{2}(\mathrm{r})\right)\right) \mathrm{dr} .
\end{aligned}
$$

This implies that

$$
\begin{aligned}
\left\|J\left(v_{1}\right)(\mathrm{t})-\mathrm{J}\left(v_{2}\right)(\mathrm{t})\right\|_{\mathrm{H}^{\mathrm{m}}\left(\mathrm{S}^{\mathfrak{n}}\right)} \leqslant & \epsilon \frac{1}{1-\gamma} \frac{1}{\epsilon} \exp \left(\frac{\gamma \mathrm{T}}{1-\gamma}\right)\left\|\int_{0}^{\mathrm{T}} \mathbf{B}_{\gamma}(\mathrm{T}-\mathrm{r})\left(\mathrm{F}\left(v_{1}(\mathrm{r})\right)-\mathrm{F}\left(v_{2}(\mathrm{r})\right)\right) \mathrm{dr}\right\|_{\mathrm{H}^{\mathrm{m}}\left(\mathrm{S}^{\mathfrak{n}}\right)} \\
& +\left\|\int_{0}^{\mathrm{t}} \mathbf{B}_{\gamma}(\mathrm{t}-\mathrm{r})\left(\mathrm{F}\left(v_{1}(\mathrm{r})\right)-\mathrm{F}\left(v_{2}(\mathrm{r})\right)\right) \mathrm{dr}\right\|_{\mathrm{H}^{\mathrm{m}}\left(\mathrm{S}^{\mathfrak{n}}\right)}=\mathcal{A}_{1}+\mathcal{A}_{2} .
\end{aligned}
$$


Let's take a look at the component $\mathcal{A}_{2}$ first. Using (3.3), we infer that

$$
\mathcal{A}_{2}=\left\|\int_{0}^{\mathrm{t}} \mathbf{B}_{\gamma}(\mathrm{t}-\mathrm{r})\left(\mathrm{F}\left(v_{1}(\mathrm{r})\right)-\mathrm{F}\left(v_{2}(\mathrm{r})\right)\right) \mathrm{dr}\right\|_{\mathrm{H}^{\mathrm{m}}\left(\mathrm{S}^{\mathfrak{n}}\right)} \leqslant \frac{1}{1-\gamma} \int_{0}^{\mathrm{t}}\left\|\mathrm{F}\left(v_{1}(\mathrm{r})\right)-\mathrm{F}\left(v_{2}(\mathrm{r})\right)\right\|_{\mathrm{H}^{\mathrm{m}-2}\left(S^{\mathrm{n}}\right)} \mathrm{dr} .
$$

Using $m \leqslant p+2$, we know that there exists $\bar{C}_{m, p}$ such that

$$
\left\|F\left(v_{1}(r)\right)-F\left(v_{2}(r)\right)\right\|_{H^{m-2}\left(S^{n}\right)} \leqslant \bar{C}_{m, p}\left\|F\left(v_{1}(r)\right)-F\left(v_{2}(r)\right)\right\|_{H^{p}\left(S^{n}\right)} \leqslant \bar{C}_{m, p} K_{f}\left\|v_{1}(r)-v_{2}(r)\right\|_{H^{m}\left(S^{n}\right)} .
$$

From the two most recent assertions, we obtain the following estimate

$$
\mathcal{A}_{2} \leqslant \frac{\overline{\mathrm{C}}_{\mathrm{m}, \mathrm{p}} \mathrm{K}_{\mathrm{f}}}{1-\gamma} \int_{0}^{\mathrm{t}}\left\|v_{1}(\mathrm{r})-v_{2}(\mathrm{r})\right\|_{\mathrm{H}^{\mathrm{m}}\left(\mathrm{S}^{\mathrm{n}}\right)} \mathrm{dr}
$$

By repeating the above demonstration, we also immediately confirm the following result

$$
\mathcal{A}_{1} \leqslant \exp \left(\frac{\gamma \mathrm{T}}{1-\gamma}\right) \frac{\overline{\mathrm{C}}_{\mathrm{m}, \mathrm{p}} \mathrm{K}_{\mathrm{f}}}{(1-\gamma)^{2}} \int_{0}^{\mathrm{T}}\left\|v_{1}(\mathrm{r})-v_{2}(\mathrm{r})\right\|_{\mathrm{H}^{\mathrm{m}}\left(\mathrm{S}^{\mathrm{n}}\right)} \mathrm{dr} \text {. }
$$

Combining (3.11), (3.12), (3.13), we find that

$$
\left\|J\left(v_{1}\right)(t)-J\left(v_{2}\right)(t)\right\|_{H^{m}\left(S^{n}\right)} \leqslant\left(\frac{\bar{C}_{m, p} K_{f}}{1-\gamma}+\exp \left(\frac{\gamma T}{1-\gamma}\right) \frac{\bar{C}_{m, p} K_{f}}{(1-\gamma)^{2}}\right) \int_{0}^{T}\left\|v_{1}(r)-v_{2}(r)\right\|_{H^{m}\left(S^{n}\right)} d r .
$$

Due to the fact that

$$
\int_{0}^{T}\left\|v_{1}(r)-v_{2}(r)\right\|_{H^{m}\left(S^{n}\right)} d r \leqslant T\left\|v_{1}-v_{2}\right\|_{L^{\infty}\left(0, T ; H^{m}\left(S^{n}\right)\right)^{\prime}}
$$

we deduce the following estimate

$$
\left\|J\left(v_{1}\right)(t)-J\left(v_{2}\right)(t)\right\|_{H^{m}\left(S^{n}\right)} \leqslant T\left(\frac{\bar{C}_{m, p} K_{f}}{1-\gamma}+\exp \left(\frac{\gamma T}{1-\gamma}\right) \frac{\bar{C}_{m, p} K_{f}}{(1-\gamma)^{2}}\right)\left\|v_{1}-v_{2}\right\|_{L^{\infty}\left(0, T ; H^{m}\left(S^{n}\right)\right)} .
$$

The right hand side of the expression (3.14) does not depend on $t$, so we have a conclusion

$$
\left\|J\left(v_{1}\right)-J\left(v_{2}\right)\right\|_{L^{\infty}\left(0, T ; H^{m}\left(S^{n}\right)\right)} \leqslant T\left(\frac{\bar{C}_{m, p} K_{f}}{1-\gamma}+\exp \left(\frac{\gamma T}{1-\gamma}\right) \frac{\bar{C}_{m, p} K_{f}}{(1-\gamma)^{2}}\right)\left\|v_{1}-v_{2}\right\|_{L^{\infty}\left(0, T_{;} ; H^{m}\left(S^{n}\right)\right)} .
$$

By choosing small $\mathrm{T}$, we can see that $\mathrm{T}\left(\frac{\overline{\mathrm{C}}_{m, p} K_{f}}{1-\gamma}+\exp \left(\frac{\gamma \mathrm{T}}{1-\gamma}\right) \frac{\overline{\mathrm{C}}_{m, p} \mathrm{~K}_{f}}{(1-\gamma)^{2}}\right)$ is less than 1. Therefore, we have immediate assertion that $J$ is the contraction mapping on the space $L^{\infty}\left(0, T ; H^{m}\left(S^{n}\right)\right)$.

Part $b$. To continue further in the demonstration, we introduce the space $L_{\theta}^{\infty}\left(0, T ; H^{\sigma}\left(S^{n}\right)\right)$, which is defined as follows

$$
\mathrm{L}_{\theta}^{\infty}\left(0, T ; H^{\sigma}\left(S^{n}\right)\right)=\left\{f:[0, T] \rightarrow \mathbb{R}, e^{-\theta t}\|f(t)\|_{H^{\sigma}\left(S^{n}\right)}<\infty\right\},
$$

where we denote the following norm

$$
\|f\|_{L_{\theta}^{\infty}\left(0, T ; H^{\sigma}\left(S^{n}\right)\right)}=\sup _{0 \leqslant t \leqslant T} e^{-\theta t}\|f(t)\|_{H^{\sigma}\left(S^{n}\right)}
$$


Let's call back the following formula

$$
\mathrm{P} w(\mathrm{t})=\mathbf{B}_{\gamma}(\mathrm{t}) \mathrm{u}_{0}+\int_{0}^{\mathrm{t}} \mathbf{B}_{\gamma}(\mathrm{t}-\mathrm{r}) \mathrm{F}(w(\mathrm{r})) \mathrm{dr} .
$$

Taking $w_{1}, w_{2} \in \mathrm{L}^{\infty}\left(0, \mathrm{~T} ; \mathrm{H}^{\mathrm{m}}\left(\mathrm{S}^{\mathrm{n}}\right)\right)$, then using a similar explanation as in (3.12), we get the following bound

$$
\begin{aligned}
e^{-\theta t}\left\|P\left(w_{1}\right)(t)-P\left(w_{2}\right)(t)\right\|_{H^{m}\left(S^{n}\right)} & \leqslant\left\|\int_{0}^{t} B_{\gamma}(t-r)\left(F\left(w_{1}(r)\right)-F\left(w_{2}(r)\right)\right) d r\right\|_{H^{m}\left(S^{n}\right)} \\
& \leqslant e^{-\theta t} \frac{\bar{C}_{m, p} K_{f}}{1-\gamma} \int_{0}^{t}\left\|w_{1}(r)-w_{2}(r)\right\|_{H^{m}\left(S^{n}\right)} d r \\
& =\frac{\bar{C}_{m, p} K_{f}}{1-\gamma} \int_{0}^{t} e^{-\theta(t-r)} e^{-\theta r}\left\|w_{1}(r)-w_{2}(r)\right\|_{H^{m}\left(S^{n}\right)} d r .
\end{aligned}
$$

It is easy to observe that

$$
\begin{aligned}
\int_{0}^{t} e^{-\theta(t-r)} e^{-\theta r}\left\|v_{1}(r)-v_{2}(r)\right\|_{H^{m}\left(S^{n}\right)} d r & \leqslant\left(\int_{0}^{t} e^{-\theta(t-r)} d r\right)\left\|w_{1}-w_{2}\right\|_{L_{\theta}^{\infty}\left(0, T_{;} ; H^{m}\left(S^{n}\right)\right)} \\
& \leqslant \frac{1}{\theta}\left\|w_{1}-w_{2}\right\|_{L_{\theta}^{\infty}\left(0, T ; H^{m}\left(S^{n}\right)\right)} .
\end{aligned}
$$

From the two most recent expressions, the following result is provided immediately

$$
e^{-\theta t}\left\|P\left(w_{1}\right)(t)-P\left(w_{2}\right)(t)\right\|_{H^{m}\left(S^{n}\right)} \leqslant \frac{\bar{C}_{m, p} K_{f}}{\theta(1-\gamma)}\left\|w_{1}-w_{2}\right\|_{L_{\theta}^{\infty}\left(0, T ; H^{m}\left(S^{n}\right)\right)} .
$$

Note that the right side of the latter expression is independent of $t$, which should lead to the following result

$$
\left\|P\left(w_{1}\right)-P\left(w_{2}\right)\right\|_{L_{\theta}^{\infty}\left(0, T ; H^{m}\left(S^{n}\right)\right)} \leqslant \frac{\bar{C}_{m, p} K_{f}}{\theta(1-\gamma)}\left\|w_{1}-w_{2}\right\|_{L_{\theta}^{\infty}\left(0, T ; H^{m}\left(S^{n}\right)\right)} .
$$

By choosing $\theta$ large enough, we get the result that the term $\frac{\overline{\mathrm{C}}_{\mathrm{m}, \mathrm{p}} \mathrm{K}_{\mathrm{f}}}{\theta(1-\gamma)}$ is less than 1 . Hence, we can deduce that $\mathrm{P}$ is a contraction on the space $\mathrm{L}_{\theta}^{\infty}\left(0, \mathrm{~T} ; \mathrm{H}^{\mathrm{m}}\left(\mathrm{S}^{\mathfrak{n}}\right)\right)$. Using Banach's co-mapping theorem, we confirm that the problem (3.1) has a unique solution in $u \in \mathrm{L}^{\infty}\left(0, \mathrm{~T} ; \mathrm{H}^{\mathrm{m}}\left(\mathrm{S}^{\mathfrak{n}}\right)\right)$.

Theorem 3.3. Let $\mathrm{F}$ be as in Theorem 3.2. Let us assume that $\mathrm{u}^{\epsilon}$ be the solution of problem (3.1). Let $\mathrm{u}$ be the solution of problem (3.1) in the case of $\epsilon=0$. Let us assume that $\mathrm{f} \in \mathrm{H}^{\mathrm{m}}\left(\mathrm{S}^{\mathrm{n}}\right)$. Then we get

$$
\left\|u^{\epsilon}(., t)-u(., t)\right\|_{H^{m}\left(S^{n}\right)} \leqslant\left(\epsilon(1-\gamma)^{-2}\|f\|_{H^{m-4}\left(S^{n}\right)}+\frac{K_{f} T \bar{C} \epsilon}{(1-\gamma)^{2}}\right) \exp \left(\frac{\bar{C}_{m, p} K_{f}}{1-\gamma} t\right) .
$$

Proof. Let us recall that $u^{\epsilon}$ be the solution of Problem (3.1) with $\epsilon>0$. Then we recall that the formula for the representation of $u^{\epsilon}$,

$$
u^{\epsilon}(x, t)=\mathbf{B}_{\gamma}(t) \mathbf{D}_{\gamma}^{\epsilon}(T) f-\epsilon \mathbf{B}_{\gamma}(t) D_{\gamma}^{\epsilon}(T) \int_{0}^{T} \mathbf{B}_{\gamma}(T-r) F\left(u^{\epsilon}(r)\right) d r+\int_{0}^{t} \mathbf{B}_{\gamma}(t-r) F\left(u^{\epsilon}(r)\right) d r .
$$

and the formula for the representation of $u$,

$$
u(x, t)=\mathbf{B}_{\gamma}(t) f+\int_{0}^{t} \mathbf{B}_{\gamma}(t-r) F(u(r)) d r .
$$


Combining two above observations, we find that the following equality

$$
\begin{aligned}
u^{\epsilon}(x, t)-u(x, t)= & \left(\mathbf{B}_{\gamma}(t) D_{\gamma}^{\epsilon}(T) f-\mathbf{B}_{\gamma}(t) f\right)+\int_{0}^{t} B_{\gamma}(t-r)\left(F\left(u^{\epsilon}(r)\right)-F(u(r))\right) d r \\
& -\epsilon \mathbf{B}_{\gamma}(t) D_{\gamma}^{\epsilon}(T) \int_{0}^{T} B_{\gamma}(T-r) F\left(u^{\epsilon}(r)\right) d r=L_{1}(x, t)+L_{2}(x, t)+L_{3}(x, t) .
\end{aligned}
$$

To evaluate the first component $L_{1}$, we have the following attention

$$
\begin{aligned}
\mathbf{D}_{\gamma}^{\epsilon}(\mathrm{T}) \nu-v & =\sum_{\ell=0}^{\infty} \sum_{k=1}^{\mathrm{N}(\mathrm{n}, \ell)}\left(\frac{1}{1+\frac{\epsilon}{1+(1-\gamma) \lambda_{\ell}} \exp \left(\frac{-\gamma \lambda_{\ell}}{1+(1-\gamma) \lambda_{\ell}} \mathrm{T}\right)} \widehat{v}_{\ell k}-\widehat{v}_{\ell k}\right) \mathrm{Y}_{\ell k}(\mathrm{x}) \\
& =\sum_{\ell=0}^{\infty} \sum_{k=1}^{\mathrm{N}(\mathrm{n}, \ell)} \frac{\frac{\epsilon}{1+(1-\gamma) \lambda_{\ell}} \exp \left(\frac{-\gamma \lambda_{\ell}}{1+(1-\gamma) \lambda_{\ell}} \mathrm{T}\right)}{1+\frac{\epsilon}{1+(1-\gamma) \lambda_{\ell}} \exp \left(\frac{-\gamma \lambda_{\ell}}{1+(1-\gamma) \lambda_{\ell}} \mathrm{T}\right)} \widehat{v}_{\ell k} \mathrm{Y}_{\ell k}(\mathrm{x}) \\
& =\epsilon \sum_{\ell=0}^{\infty} \sum_{k=1}^{\mathrm{N}(\mathrm{n}, \ell)} \frac{\exp \left(\frac{-\gamma \lambda_{\ell}}{1+(1-\gamma) \lambda_{\ell}} \mathrm{T}\right)}{1+(1-\gamma) \lambda_{\ell}+\epsilon \exp \left(\frac{-\gamma \lambda_{\ell}}{1+(1-\gamma) \lambda_{\ell}} \mathrm{T}\right)} \widehat{v}_{\ell k} \mathrm{Y}_{\ell k}(\mathrm{x})
\end{aligned}
$$

where we have used the definition of (3.8). If $v \in \mathrm{H}^{\sigma}\left(\mathrm{S}^{\mathrm{n}}\right)$, then we get the following bound

$$
\begin{aligned}
\left\|\mathbf{D}_{\gamma}^{\epsilon}(\mathrm{T}) \nu-v\right\|_{\mathrm{H}^{\sigma}\left(\mathrm{S}^{n}\right)}^{2} & =\epsilon^{2} \sum_{\ell=0}^{\infty} \sum_{k=1}^{N(n, \ell)} \lambda_{\ell}^{\sigma}\left(\frac{\exp \left(\frac{-\gamma \lambda_{\ell}}{1+(1-\gamma) \lambda_{\ell}} \mathrm{T}\right)}{1+(1-\gamma) \lambda_{\ell}+\epsilon \exp \left(\frac{-\gamma \lambda_{\ell}}{1+(1-\gamma) \lambda_{\ell}} \mathrm{T}\right)}\right)^{2}\left|\widehat{v}_{\ell k}\right|^{2} \\
& \leqslant \epsilon^{2}(1-\gamma)^{-2} \sum_{\ell=0}^{\infty} \sum_{k=1}^{N(n, \ell)} \lambda_{\ell}^{\sigma-2}\left|\widehat{v}_{\ell k}\right|^{2},
\end{aligned}
$$

which allows us to obtain that

$$
\left\|\mathbf{D}_{\gamma}^{\epsilon}(\mathbf{T}) \nu-v\right\|_{H^{\sigma}\left(S^{n}\right)} \leqslant \epsilon(1-\gamma)^{-1}\|v\|_{H^{\sigma-2}\left(S^{n}\right)} .
$$

By looking at the estimate (3.3), it follows from (3.17) that

$$
\begin{aligned}
\left\|\mathrm{L}_{1}(., \mathrm{t})\right\|_{\mathrm{H}^{\mathrm{m}}\left(\mathrm{S}^{\mathfrak{n}}\right)} & =\left\|\mathbf{B}_{\gamma}(\mathrm{t}) \mathbf{D}_{\gamma}^{\epsilon}(\mathrm{T}) \mathrm{f}-\mathbf{B}_{\gamma}(\mathrm{t}) \mathrm{f}\right\|_{\mathrm{H}^{\mathrm{m}}\left(\mathrm{S}^{\mathfrak{n}}\right)} \\
& \leqslant \frac{1}{1-\gamma}\left\|\mathbf{D}_{\gamma}^{\epsilon}(\mathrm{T}) \mathrm{f}-\mathrm{f}\right\|_{\mathrm{H}^{\mathrm{m}-2}\left(\mathrm{~S}^{\mathfrak{n}}\right)} \leqslant \epsilon(1-\gamma)^{-2}\|\mathrm{f}\|_{\mathrm{H}^{\mathrm{m}-4}\left(S^{\mathfrak{n}}\right)} .
\end{aligned}
$$

Next, we continue to treat the second term $\mathrm{L}_{2}$. If $v \in \mathrm{H}^{\sigma}\left(\mathrm{S}^{\mathfrak{n}}\right)$, then we note the following estimate

$$
\begin{aligned}
\left\|\mathbf{D}_{\gamma}^{\mathrm{\epsilon}}(\mathrm{T}) \nu\right\|_{H^{\sigma}\left(S^{n}\right)}^{2} & =\sum_{l=0}^{\infty} \sum_{k=1}^{N(n, \ell)}\left(1+\lambda_{\ell}\right)^{\sigma}\left(\frac{1}{1+\frac{\epsilon}{1+(1-\gamma) \lambda_{\ell}} \exp \left(\frac{-\gamma \lambda_{\ell}}{1+(1-\gamma) \lambda_{\ell}} \mathrm{T}\right)}\right)^{2}\left|\hat{v}_{\ell k}\right|^{2} \\
& \leqslant \sum_{\ell=0}^{\infty} \sum_{k=1}^{N(n, \ell)}\left(1+\lambda_{\ell}\right)^{\sigma}\left|\widehat{v}_{\ell k}\right|^{2}=\|v\|_{H^{\sigma}\left(S^{n}\right)}^{2} .
\end{aligned}
$$

Using (3.3), we get the following bound

$$
\left\|\mathbf{B}_{\gamma}(\mathrm{t}) \mathbf{D}_{\gamma}^{\epsilon}(\mathbf{T}) v\right\|_{H^{\sigma}\left(S^{n}\right)} \leqslant \frac{1}{1-\gamma}\left\|\mathbf{D}_{\gamma}^{\epsilon}(T) v\right\|_{H^{\sigma-2}\left(S^{n}\right)} \leqslant \frac{1}{1-\gamma}\|v\|_{H^{\sigma-2}\left(S^{n}\right)} .
$$


Hence, we obtain that

$$
\begin{aligned}
\left\|-\epsilon \mathbf{B}_{\gamma}(t) \mathbf{D}_{\gamma}^{\epsilon}(T) \int_{0}^{T} \mathbf{B}_{\gamma}(T-r) F\left(u^{\epsilon}(r)\right) d r\right\|_{H^{m}\left(S^{n}\right)} & \leqslant \frac{\epsilon}{1-\gamma}\left\|\int_{0}^{T} \mathbf{B}_{\gamma}(T-r) F\left(u^{\epsilon}(r)\right) d r\right\|_{H^{m-2}\left(S^{n}\right)} \\
& \leqslant \frac{\epsilon}{(1-\gamma)^{2}} \int_{0}^{T}\left\|F\left(u^{\epsilon}(r)\right) d r\right\|_{H^{m-2}\left(S^{n}\right)} \\
& \leqslant \frac{K_{f} \epsilon}{(1-\gamma)^{2}} \int_{0}^{T}\left\|u^{\epsilon}(., r) d r\right\|_{H^{m}\left(S^{n}\right)^{\prime}}
\end{aligned}
$$

where we have used the fact that

$$
\left\|F\left(u^{\epsilon}(r)\right) d r\right\|_{H^{m-2}\left(S^{n}\right)} \leqslant K_{f}\left\|u^{\epsilon}(., r) d r\right\|_{H^{m}\left(S^{n}\right)} .
$$

It is noted that from Theorem 3.2, we can deduce that

$$
\left\|u^{\epsilon}\right\|_{L^{\infty}\left(0, T ; H^{m}\left(S^{n}\right)\right)} \leqslant \bar{C},
$$

where $\bar{C}$ is independent of $\epsilon$. Therefore, we infer that

$$
\left\|\mathrm{L}_{2}(., \mathrm{t})\right\|_{\mathrm{H}^{\mathrm{m}}\left(\mathrm{S}^{\mathrm{n}}\right)} \leqslant \frac{\mathrm{K}_{\mathrm{f}} \mathrm{T} \overline{\mathrm{C}} \epsilon}{(1-\gamma)^{2}}
$$

By a similar method as in (3.12), we also obtain the following estimate

$$
\left\|\mathrm{L}_{3}(., \mathrm{t})\right\|_{H^{\mathrm{m}}\left(S^{n}\right)} \leqslant \frac{\overline{\mathrm{C}}_{\mathrm{m}, \mathrm{p}} \mathrm{K}_{\mathrm{f}}}{1-\gamma} \int_{0}^{\mathrm{t}}\left\|\mathrm{u}^{\epsilon}(., \mathrm{r})-\mathrm{u}(., r)\right\|_{H^{\mathrm{m}}\left(S^{n}\right)} \mathrm{dr}
$$

Combining (3.16), (3.18), and (3.19), we infer that

$$
\begin{aligned}
\left\|u^{\epsilon}(., t)-u(., t)\right\|_{H^{m}\left(S^{n}\right)} & \leqslant\left\|L_{1}(., t)\right\|_{H^{m}\left(S^{n}\right)}+\left\|L_{2}(., t)\right\|_{H^{m}\left(S^{n}\right)}+\left\|L_{3}(., t)\right\|_{H^{m}\left(S^{n}\right)} \\
& \leqslant \epsilon(1-\gamma)^{-2}\|f\|_{H^{m-4}\left(S^{n}\right)}+\frac{K_{f} T \bar{C} \epsilon}{(1-\gamma)^{2}}+\frac{\bar{C}_{m, p} K_{f}}{1-\gamma} \int_{0}^{t}\left\|u^{\epsilon}(., r)-u(., r)\right\|_{H^{m}\left(S^{n}\right)} d r .
\end{aligned}
$$

By applying Gronwall's inequality, we can deduce the desired result (3.15).

\section{Conclusion}

In this paper, we study the parabolic problem associated with non-local conditions, with the CaputoFabrizio derivative for equations on the sphere. We prove that the problem has only the solution for two cases. In case $\epsilon=0$, we prove the problem has a local solution. In case $\epsilon>0$, then the problem has a global solution. The main tools is the Banach's fixed point theorem, and some Fourier series analysis involved some evaluation of spherical harmonic function.

\section{Acknowledgment}

This research is funded by Thu Dau Mot University, Binh Duong Province, Vietnam under grant number DT.21.1- 013. 


\section{References}

[1] A. Abdelouaheb, Asymptotic stability in Caputo-Hadamard fractional dynamic equation, Results Nonlinear Anal., 4 (2021), 77-86. 1

[2] K. A. Abro, A. Atangana, Mathematical analysis of memristor through fractal-fractional differential operators: a numerical study, Math. Methods Appl. Sci., 43 (2020), 6378-6395. 1

[3] B. Acay, M. Inc, Fractional modeling of temperature dynamics of a building with singular kernels, Chaos Solitons Fractals, 142 (2021), 9 pages. 1

[4] T. M. Atanacković, S. Pillipović, D. Zorica, Properties of the Caputo-Fabrizio fractional derivative and its distributional settings, Fract. Calc. Appl. Anal., 21 (2018), 29-44. 1

[5] A. Atangana, A. Akgül, K. M. Owolabi, Analysis of fractal fractional differential equations, Alexandria Eng. J., 59 (2020), 1117-1134. 1

[6] A. Atangana, E. Bonyah, Fractional stochastic modeling: New approach to capture more heterogeneity, Chaos, 29 (2019), 13 pages.

[7] A. Atangana, Z. Hammouch Fractional calculus with power law: The cradle of our ancestors, Eur. Phys. J. Plus, 134 (2019), 13 pages. 1

[8] Z. Brzeźniak, B. Goldys, Q. T. Le Gia, Random attractors for the stochastic Navier-Stokes equations on the 2D unit sphere, J. Math. Fluid Mech., 20 (2018), 227-253. 1

[9] M. Caputo, M. Fabrizio, A new definition of fractional derivative without singular kernel, Progr. Fract. Differ. Appl., 2 (2015), 1-13. 1, 1

[10] M. Caputo, M. Fabrizio, Applications of new time and spatial fractional derivatives with exponential kernels, Progr. Fract. Differ. Appl., 2 (2016), 1-11. 1

[11] M. A. Dokuyucu, Caputo and Atangana-Baleanu-Caputo Fractional Derivative Applied to Garden Equation, Turkish J. Sci., 5 (2020), 1-7. 1

[12] M. A. Dokuyucu, Analysis of a novel finance chaotic model via ABC fractional derivative, Numer. Methods Partial Differ. Equ., 37 (2021), 1583-1590.

[13] M. A. Dokuyucu, E. Çelik, Analyzing a novel coronavirus model (Covid-19) in the sense of Caputo Fabrizio fractional operator, Appl. Comput. Math., 20, (2021), 49-69.

[14] M. A. Dokuyucu, H. Dutta, C. Yildirim, Application of non-local and non-singular kernel to an epidemiological model with fractional order, Math. Methods Appl. Sci., 44 (2021), 3468-3484. 1

[15] R. M. Ganji, H. Jafari, S. Nemati, A new approach for solving integro-differential equations of variable order, J. Comput. Appl. Math., 379 (2020), 13 pages. 1

[16] H. Günerhan, H. Dutta, M. A. Dokuyucu, W. Adel, Analysis of a fractional HIV model with Caputo and constant proportional Caputo operators, Chaos Solitons Fractals, 139 (2020), 19 pages. 1

[17] Z. Hammouch, T. Mekkaoui, P. Agarwal, Optical solitons for the Calogero-Bogoyavlenskii-Schiff equation in (2+ 1) dimensions with time-fractional conformable derivative, Eur. Phys. J., 133 (2018), 1-6. 1

[18] N. N. Hung, H. D. Binh, N. H. Luc, N. T. K. An, L. D. Long, Stochastic sub-diffusion equation with conformable derivative driven by standard Brownian motion, Adv. Theory Nonlinear Anal. Appl., 5 (2021), 287-299. 1

[19] H. Jafari, H. Tajadodi, R. M. Ganji, A numerical approach for solving variable order differential equations based on Bernstein polynomials, Comput. Math. Methods, 1 (2019), 11 pages. 1

[20] F. Jarad, T. Abdeljawad, Z. Hammouch, On a class of ordinary differential equations in the frame of Atangana-Baleanu fractional derivative, Chaos Solitons Fractals, 117 (2018), 16-20. 1

[21] M. M. Khader, K. M. Saad, Z. Hammouch, D. Baleanu, A spectral collocation method for solving fractional KdV and KdV-Burgers equations with non-singular kernel derivatives, Appl. Numer. Math., 161 (2021), 137-146. 1

[22] I. Koca, E. Akcetin, P. Yaprakdal, Numerical approximation for the spread of SIQR model with Caputo fractional order derivative, Turkish J. Sci., 5 (2020), 124-139. 1

[23] Z. Korpinar, M. Inc, M. Bayram, Theory and application for the system of fractional Burger equations with Mittag leffler kernel, Appl. Math. Comput., 367 (2020), 11 pages. 1

[24] S. Kumar, A. Atangana, A numerical study of the nonlinear fractional mathematical model of tumor cells in presence of chemotherapeutic treatment, Int. J. Biomath., 13 (2020), 17 pages. 1

[25] Q. T. Le Gia, Galerkin approximation of elliptic PDEs on spheres, J. Approx. Theory, 130 (2004), 125-149. 1

[26] Q. T. Le Gia, Approximation of parabolic PDEs on spheres using collocation method, Adv. Comput. Math., 22 (2005), 377-397.

[27] Q. T. Le Gia, I. H. Sloan, T. Tran, Overlapping additive Schwarz preconditioners for elliptic PDEs on the unit sphere, Math. Comp., 78 (2009), 79-101.

[28] Q. T. Le Gia, N. H. Tuan, T. Tran, Solving the backward heat equation on the unit sphere, ANZIAM J. Electron. Suppl., 56 (2014), 262-278. 1

[29] J. Losada, J. J. Nieto, Properties of a new fractional derivative without singular kernel, Prog. Fract. Differ. Appl., 1 (2015), 87-92. 1

[30] N. H. Luc, H. Jafari, P. Kumam, N. H. Tuan, On an initial value problem for time fractional pseudo-parabolic equation with Caputo derivarive, Mathematical Methods in the Applied Sciences, (2021). 1 
[31] B. D. Nghia, N. H. Luc, H. D. Binh, L. D. Long, Regularization method for the problem of determining the source function using integral conditions, Adv. Theor. Nonlinear Anal. Appl., 5 (2021), 351-362. 1

[32] O. Nikan, H. Jafari, A. Golbabai, Numerical analysis of the fractional evolution model for heat flow in materials with memory, Alexandria Eng. J., 59 (2020), 2627-2637. 1

[33] N. D. Phuong, N. H. Luc, Note on a Nonlocal Pseudo-Parabolic Equation on the Unit Sphere, Dyn. Syst. Appl., 30 (2021), 295-304. 1

[34] N. H. Sweilam, S. M. Al-Mekhlafi, T. Assiri, A. Atangana, Optimal control for cancer treatment mathematical model using Atangana-Baleanu-Caputo fractional derivative, Adv. Difference Equ., 2020 (2020), 21 pages. 1

[35] N. H. Tuan, L. N. Huynh, T. B. Ngoc, Y. Zhou, On a backward problem for nonlinear fractional diffusion equations, Appl. Math. Lett., 92 (2019), 76-84. 1

[36] A. Yusuf, B. Acay, U. T. Mustapha, M. Inc, D. Baleanu, Mathematical modeling of pine wilt disease with Caputo fractional operator, Chaos Solitons Fractals, 14 (2011), 13 pages. 1 